\title{
Electrocardiographic and Echocardiographic Insights From a Prospective Registry of Asian Elite Athletes
}

OPEN ACCESS

Edited by:

Sabina Gallina,

University of Studies G. d'Annunzio

Chieti and Pescara, Italy

Reviewed by:

Frédéric Schnell,

Centre Hospitalier Universitaire (CHU) de Rennes, France

Roman Leischik

Witten/Herdecke University, Germany

Alessandro Zorzi,

University Hospital of Padua, Italy

${ }^{*}$ Correspondence:

Tee Joo Yeo

tee_joo_yeo@nuhs.edu.sg

Specialty section:

This article was submitted to Sex and Gender in Cardiovascular

Medicine,

a section of the journa

Frontiers in Cardiovascular Medicine

Received: 21 October 2021

Accepted: 29 November 2021

Published: 03 January 2022

Citation:

Yeo TJ, Wang M, Grignani R,

McKinney J, Koh LP, Tan FHY, Chan GCT, Tay N, Chan S-P, Lee C-H,

Oxborough D, Malhotra A, Sharma $S$

and Richards AM (2022)

Electrocardiographic and

Echocardiographic Insights From a

Prospective Registry of Asian Elite

Athletes.

Front. Cardiovasc. Med. 8:799129.

doi: 10.3389/fCvm.2021.799129
Tee Joo Yeo ${ }^{1,2 *}$, Mingchang Wang ${ }^{3}$, Robert Grignani ${ }^{4}$, James McKinney ${ }^{5}$, Lay Pheng Koh ${ }^{1}$, Frankie Hun Yau Tan ${ }^{6,7}$, Gregory Chung Tsing Chan ${ }^{6}$, Nigel Tay ${ }^{8}$, Siew-Pang Chan ${ }^{1,2}$, Chi-Hang Lee ${ }^{1,2}$, David Oxborough ${ }^{9}$, Aneil Malhotra ${ }^{10,11}$, Sanjay Sharma ${ }^{12}$ and Arthur Mark Richards ${ }^{1,2}$

${ }^{1}$ Cardiac Department, National University Heart Centre Singapore, Singapore, Singapore, ${ }^{2}$ Cardiovascular Research Institute, National University Heart Centre Singapore, Singapore, Singapore, ${ }^{3}$ National University Hospital Sports Centre, National University Hospital, Singapore, Singapore, ${ }^{4}$ Department of Paediatrics, National University Hospital, Singapore, Singapore, ${ }^{5}$ SportsCardiologyBC, University of British Columbia, Vancouver, BC, Canada, ${ }^{6}$ Sport Science and Medicine Centre, Singapore Sport Institute, Sport Singapore, Singapore, Singapore, ${ }^{7}$ Department of Physiology, Yong Loo Lin School of Medicine, National University of Singapore, Singapore, Singapore, ${ }^{8}$ Family Medicine Department, Cavendish Doctors, Auckland, New Zealand, ${ }^{9}$ Sport and Exercise Sciences, Liverpool John Moores University, Liverpool, United Kingdom,

${ }^{10}$ Division of Cardiovascular Sciences, University of Manchester, Manchester NHS Foundation Trust, Manchester, United Kingdom, ${ }^{11}$ Manchester Institute of Health and Performance, Manchester, United Kingdom, ${ }^{12}$ Cardiology Clinical Academic Group, St George's University of London, London, United Kingdom

Background: Asian representation in sport is increasing, yet there remains a lack of reference values for the Asian athlete's heart. Consequently, current guidelines for cardiovascular screening recommend using Caucasian athletes' norms to evaluate Asian athletes. This study aims to outline electrocardiographic and echocardiographic characteristics of the Asian athlete's heart using a Singaporean prospective registry of Southeast (SE) Asian athletes.

Methods and Results: One hundred and fifty elite athletes, mean age of $26.1 \pm$ 5.7 years (50\% males, 88\% Chinese), were evaluated using a questionnaire, 12-lead electrocardiogram (ECG) and transthoracic echocardiogram. All ECGs were analyzed using the 2017 International Recommendations. Echocardiographic data were presented by gender and sporting discipline. The prevalence of abnormal ECGs among SE Asian athletes was 6.7\% - higher than reported figures for Caucasian athletes. The abnormal ECGs comprised mainly anterior T wave inversions (ATWI) beyond lead V2, predominantly in female athletes from mixed/endurance sport (9.3\% prevalence amongst females). None had echocardiographic structural abnormalities. Male athletes had reduced global longitudinal strain compared to females $(-18.7 \pm 1.6$ vs. $-20.7 \pm 2.1 \%, p<0.001)$. Overall, SE Asian athletes had smaller left ventricular cavity sizes and wall thickness compared to non-Asian athletes.

Conclusion: SE Asian athletes have higher abnormal ECG rates compared to Caucasian athletes, and also demonstrate structural differences that should be accounted for when interpreting their echocardiograms compared to athletes of other ethnicities.

Keywords: athlete's heart, sports cardiology, registry, Asian athlete, cardiac remodeling 


\section{INTRODUCTION}

Asian athletes feature prominently in international competitive sport and continue to show improvement at the pinnacle of physical ability-the Olympic Games (1). Altogether, 48 countries combine to form Asia, the largest and most populous continent in the world with a total population exceeding 4.4 billion (2). Despite this enormous population base, there remains a dearth of data for the Asian athlete's ECG and echocardiogram.

The electrocardiogram (ECG) is instrumental in differentiating pathology from physiology in the athlete's heart (3). ECG screening in athletes over the past decade has seen the development of qualitative and quantitative cutoffs, as well as ethnicity-specific recommendations. For instance, anterior T wave inversions (ATWI) up to lead V2 are deemed physiological in Caucasian athletes, whereas in Black athletes, ATWI up to lead V4 are considered within normal limits when preceded by J-point elevation and convex ST segment elevation. These ECG recommendations have reduced false positive rates substantially while preserving sensitivity in identifying pathology in athletes (4). In parallel to the ECG, echocardiographic imaging of Caucasian and Black athletes has also progressed considerably, leading to the establishment of normal reference values (5).

Physiological ECG and echocardiographic limits for Asian athletes have yet to be defined, with current guidelines recommending using Caucasian athletes' norms to evaluate Asian athletes (6). This study aims to highlight ECG and echocardiographic characteristics for Asian athletes via a prospective registry of athletes from Southeast (SE) Asia. Singapore, an island city-state with a multi-ethnic SE Asian population of Chinese (74.1\%), Malay (13.4\%), Indian (9.2\%), and others (3.3\%), forms the base for this study (7).

\section{METHODS}

The Singapore Sports Cardiology Registry is a descriptive, cross-sectional, and prospective registry of active elite athletes aged $\geq 18$ years representing the country in competitive sport regardless of sporting discipline. The study was carried out between January and October 2018 in the Singapore Sport Institute Sports Medicine Centre, which is the only ambulatory centre in the country that provides medical assessment and clearance for all national athletes prior to sport participation. One hundred and fifty consecutive elite athletes from 32 different sporting disciplines completed a questionnaire where data collected included: age, gender, ethnicity, sporting discipline, training history, medical history, and family history of sudden cardiac death.

\section{Electrocardiography}

Standard resting 12-lead ECGs were performed on athletes in a supine position, using an ELI 230 ECG machine (Mortara, Milwaukee, WI) at a paper speed of $25 \mathrm{~mm} / \mathrm{s}$. All ECGs were analyzed in the digital unfiltered format for abnormalities based on established ECG interpretation criteria for athletes, namely the European Society of Cardiology recommendations in 2010 (ESC2010), the Seattle Criteria in 2013 (SC2013), the Refined Criteria in 2014 (RC2014) and the International recommendations in 2017 (IR2017) (8-11).

\section{Echocardiography}

All recruited athletes underwent resting M-mode, twodimensional (2D) and Doppler transthoracic echocardiography using a Vivid S6 ultrasound system (GE Healthcare, Milwaukee, WI), in accordance with American and British Society of Echocardiography guidelines (12-14). All echocardiograms were performed immediately after the resting 12-lead ECG. Left ventricular (LV) ejection fraction was measured using the biplane method of disks. Pulsed wave tissue doppler imaging of the septal, lateral LV and tricuspid annuli was performed in the apical 4-chamber view to obtain peak early $\left(\mathrm{e}^{\prime}\right)$ and late $\left(\mathrm{a}^{\prime}\right)$ myocardial velocities and the ratio of early diastolic transmitral flow velocity to $\mathrm{e}^{\prime}\left(\mathrm{E} / \mathrm{e}^{\prime}\right)$.

Radiofrequency data from three cardiac cycles were stored and indices of myocardial deformation obtained by speckle-tracking analysis using EchoPac software (Version 11.1.8, GE Healthcare, Horten, Norway). Automated functional imaging was utilized to track and analyse peak systolic strain using a tri-plane imaging probe. Global longitudinal strain (averaged) was measured for all participants. Measures of right ventricular systolic function, 2D fractional area change and tricuspid annular plane systolic excursion (TAPSE), were also obtained.

LV geometry was classified into four groups based on American and European Society of Echocardiography guidelines: normal geometry, concentric remodeling, eccentric hypertrophy, and concentric hypertrophy $(12,14)$. This was based on cutoffs for relative wall thickness (abnormal $>0.42$ ) and LV mass index (LV hypertrophy defined as LV mass index $>95 \mathrm{~g} / \mathrm{m}^{2}$ in females and $>115 \mathrm{~g} / \mathrm{m}^{2}$ in males).

All echocardiographic images were separately reviewed and analyzed by two independent cardiologists, blinded to the participants' ethnicity, sporting discipline, and training volume. Discrepancies in quantitative parameters were averaged and presented as mean values, whereas mutual discussion was carried out for qualitative differences.

\section{Statistical Analysis}

The sample characteristics were presented as mean \pm standard deviation (SD) or frequency (\%), depending on their nature. The ECGs and echocardiographic data were presented by gender as well as sport discipline. Sport disciplines, based on the European Association of Preventive Cardiology (EAPC) position statement in 2017, were divided into two groups-skill and power (low to moderate oxygen consumption) vs. mixed and endurance (moderate to high oxygen consumption) (5). Independent $t$ tests were performed to ascertain if there were significant differences in ECGs and echocardiographic parameters between the groups. Regression analyses were carried out to examine the association of demographics (i.e., age, gender, ethnicity), sporting discipline and training hours on ECG abnormalities as well as commonly utilized echocardiographic parameters. Data analysis was performed with STATA version 14 (Statacorp, Texas) and all statistical tests were conducted with $5 \%$ level of significance. 
Written informed consent was obtained from all participants. All study procedures conformed to the ethical guidelines of the 1975 Declaration of Helsinki.

\section{RESULTS}

\section{Baseline Characteristics}

Of the 150 SE Asian athletes, there were 75 (50\%) males, and the ethnic distribution was Chinese (88\%), Malay (5.3\%), Indian (3.3\%), and others [Sikh, Indian-Chinese and Indonesian] (3.3\%). These are reflective of ethnic groups found within SE Asia. Participants had a mean age of $26.1 \pm 5.7$ years. Their average training duration was $19.3 \pm 8.8 \mathrm{~h}$ per week, with mean competitive experience of $8.5 \pm 4.7$ years (Table 1).

TABLE 1 | Baseline characteristics and sport classification of athletes.

\begin{tabular}{lc}
\hline & Mean \pm SD/Frequency (\%) \\
\hline Age (years) & $26.1 \pm 5.7$ \\
Male gender & $75(50)$ \\
Ethnic group & \\
- Chinese & $132(88)$ \\
- Malay & $8(5.3)$ \\
- Indian & $5(3.3)$ \\
- Others & $5(3.3)$ \\
Body mass index $\left(\mathrm{kg} / \mathrm{m}^{2}\right)$ & $22.9 \pm 3.1$ \\
Body surface area $\left(\mathrm{m}^{2}\right)$ & $1.8 \pm 0.2$ \\
Training hours/week & $19.3 \pm 8.8$ \\
Number of years competing & $8.5 \pm 4.7$ \\
EAPC sport category & \\
- Skill & $20(13.3)$ \\
- Power & $24(16)$ \\
- Mixed & $50(33.3)$ \\
- Endurance & $56(37.3)$
\end{tabular}

Baseline demographics between Chinese and non-Chinese athletes were comparable.

\section{Electrocardiographic Data}

The most common training-related changes based on IR2017 were: sinus bradycardia $(65.3 \%)$, early repolarization $(46 \%)$, and sinus arrhythmia (25.3\%). Early repolarization (70.7 vs. $21.3 \%, p<0.0001)$ and voltage criteria for LV hypertrophy (17.3 vs. $4 \%, p<0.02$ ) were more common in males than females. Athletes in mixed and endurance sports demonstrated more sinus bradycardia ( 73.6 vs. $45.4 \%, p=0.001$ ), incomplete right bundle branch block ( 15.1 vs. $2.3 \%, p=0.024)$, early repolarization ( 53.8 vs. $27.3 \%, p=0.004)$ and voltage criteria for LV hypertrophy (15.8 vs. $0 \%, p=0.003$ ) compared to those in skill and power-based sports. Borderline findings based on IR2017 were noted in 3 separate athletes -2 with left atrial enlargement and 1 with complete right bundle branch block.

Using ESC2010, 31 ECGs in our cohort were identified with abnormal findings unrelated to training $(20.7 \%$ prevalence), and application of SC2013 reduced the number of abnormal ECGs to 13 (8.7\% prevalence). Identical results for both RC2014 and IR2017 were obtained $(6.7 \%$ prevalence). There were no differences in prevalence of abnormal ECGs between Chinese and non-Chinese athletes.

Characteristics of all 10 athletes with abnormal ECGs based on IR2017 are outlined in Table 2, and digital ECGs are available as Supplementary Material. All were Chinese and engaged in mixed or endurance sports. Of these, 8 athletes (7 females; 9.3\% prevalence among female athletes) showed anterior $\mathrm{T}$ wave inversions (ATWI) in 2 contiguous leads beyond V1. Echocardiograms did not reveal any structural abnormalities in all 10 athletes apart from LV hypertrophy. Of 4 athletes who consented to undergo cardiac magnetic resonance imaging, no abnormalities were found.

TABLE 2 | Characteristics of athletes with abnormal electrocardiograms based on the 2017 International Recommendations.

\begin{tabular}{|c|c|c|c|c|c|c|c|c|c|c|c|c|}
\hline No & $\begin{array}{l}\text { Age } \\
\text { (yrs) }\end{array}$ & Gender & Ethnicity & BSA $\left(m^{2}\right)$ & Sport & $\begin{array}{c}\text { Weekly training } \\
\text { hours }\end{array}$ & ECG abnormality & $\begin{array}{l}\text { LVWT } \\
\text { (mm) }\end{array}$ & $\begin{array}{l}\text { LVIDD } \\
(\mathrm{mm})\end{array}$ & $\begin{array}{l}\text { LV mass } \\
\quad(g)\end{array}$ & $\begin{array}{l}\text { LVEF } \\
(\%)\end{array}$ & GLS (\%) \\
\hline 1 & 23.4 & Male & Chinese & 1.87 & Kayak & 25 & QRS $140 \mathrm{msec}$ & 12 & 55 & 288.9 & 58 & -18 \\
\hline 3 & 18.0 & Female & Chinese & 1.58 & Tennis & 27 & ATWI V1-V3 & 9 & 44 & 148.7 & 58 & -19 \\
\hline $4^{\star}$ & 36.2 & Female & Chinese & 1.44 & Marathon & 10 & ATWI V1-V4 & 8 & 48 & 132.4 & 60 & -20 \\
\hline 7 & 32.2 & Female & Chinese & 1.72 & Netball & 10 & ATWI V1-V3 & 7 & 47 & 108.8 & 53 & -20 \\
\hline 8 & 33.1 & Female & Chinese & 1.49 & Marathon & 10 & ATWI V1-V3 & 7 & 48 & 111.3 & 63 & -23 \\
\hline $9^{*}$ & 38.8 & Male & Chinese & 1.47 & Marathon & 12 & ATWI V1-V3 & 10 & 52 & 185.7 & 59 & -20 \\
\hline 10 & 26.7 & Female & Chinese & 1.52 & Dragonboat & 22 & ATWI V1-V3 & 7 & 46 & 105.9 & 63 & -23 \\
\hline
\end{tabular}

BSA, body surface area; ECG, electrocardiogram; LVWT, Left ventricular wall thickness; LVIDD, Left ventricular internal diameter in diastole; LVEF, Left ventricular ejection fraction; GLS, global longitudinal strain; PVC, premature ventricular contraction; ATWI, Anterior T wave inversion.

${ }^{*}$ Athletes with cardiac magnetic resonance imaging performed - no pathology detected. 
TABLE 3 | Gender differences in echocardiographic parameters for Singapore athletes.

\begin{tabular}{|c|c|c|c|c|c|c|c|}
\hline & \multicolumn{3}{|c|}{ Male $(n=75)$} & \multicolumn{3}{|c|}{ Female $(n=75)$} & \multirow[t]{2}{*}{$P$} \\
\hline & Mean & SD & Min, Max & Mean & SD & Min, Max & \\
\hline \multicolumn{8}{|l|}{ Left heart } \\
\hline LVWT (mm) & 9.4 & 1.2 & 7,13 & 7.7 & 1.1 & 5,11 & $<0.001$ \\
\hline LVWT/BSA (mm/m²) & 5.1 & 0.7 & $3.6,7.0$ & 4.7 & 0.8 & $3.0,7.2$ & 0.002 \\
\hline LVIDD (mm) & 51.6 & 3.7 & 43,63 & 47.7 & 3.6 & 40,57 & $<0.001$ \\
\hline LVIDD/BSA $\left(\mathrm{mm} / \mathrm{m}^{2}\right)$ & 27.6 & 2.4 & 23,35 & 29.0 & 2.7 & 21,34 & 0.001 \\
\hline LVPWD (mm) & 9.6 & 1.3 & 6,13 & 7.9 & 1.2 & 5,11 & $<0.001$ \\
\hline Relative wall thickness & 0.37 & 0.05 & $0.24,0.47$ & 0.33 & 0.05 & $0.2,0.5$ & $<0.001$ \\
\hline LV mass (g) & 181.9 & 43.7 & $103,316.2$ & 122.1 & 29.3 & $64.5,199.6$ & $<0.001$ \\
\hline LV mass/BSA $\left(\mathrm{g} / \mathrm{m}^{2}\right)$ & 97.0 & 22.5 & 62,160 & 74.2 & 17.7 & $39.3,129.6$ & $<0.001$ \\
\hline LVEDV (ml) & 127.4 & 22.1 & 77,189 & 94.2 & 20.1 & 60,154 & $<0.001$ \\
\hline LVEDV/BSA $\left(\mathrm{ml} / \mathrm{m}^{2}\right)$ & 68.1 & 11.7 & 97.7 & 56.9 & 10.9 & 83.2 & $<0.001$ \\
\hline LV stroke volume (ml) & 74.1 & 14.2 & 42,117 & 56.2 & 12.4 & 33,94 & $<0.001$ \\
\hline LVEF (\%) & 58.2 & 4.1 & 47,67 & 60.1 & 4.0 & 46,67 & 0.004 \\
\hline LA (mm) & 36.1 & 4.6 & 25,48 & 33.0 & 3.7 & 25,43 & $<0.001$ \\
\hline LA/BSA $\left(\mathrm{mm} / \mathrm{m}^{2}\right)$ & 19.3 & 2.6 & $14.6,27.2$ & 20.0 & 2.2 & $15.6,26.5$ & NS \\
\hline LA volume (ml) & 65.4 & 19.0 & 36,135 & 53.0 & 14.1 & 28,96 & $<0.001$ \\
\hline LA volume/BSA (ml/m²) & 34.9 & 9.9 & $19.6,70.6$ & 32.1 & 7.8 & $17.2,48.8$ & NS \\
\hline E velocity $(\mathrm{cm} / \mathrm{s})$ & 74.3 & 14.8 & 23,106 & 84.6 & 15.1 & 55,123 & $<0.001$ \\
\hline DT (ms) & 157.3 & 19.9 & 118,208 & 149.8 & 19.4 & 106,198 & 0.02 \\
\hline A velocity (cm/s) & 39.8 & 8.0 & 21,60 & 43.0 & 9.8 & 25,69 & 0.03 \\
\hline $\mathrm{E} / \mathrm{A}$ & 1.9 & 0.5 & $0.5,3.9$ & 2.0 & 0.5 & $1.3,4.2$ & NS \\
\hline Septal annular E' (cm/s) & 11.8 & 1.8 & 7,16 & 11.9 & 1.8 & 8,17 & NS \\
\hline Septal annular A' (cm/s) & 7.2 & 1.5 & 4,11 & 6.4 & 1.1 & 4,9 & 0.001 \\
\hline Septal annular E'/A' & 1.7 & 0.5 & $0.7,3.5$ & 1.9 & 0.4 & $1.1,3$ & 0.03 \\
\hline Septal annular E/E' & 6.3 & 1.1 & $3.3,8.8$ & 7.3 & 1.5 & $4.1,10.9$ & $<0.001$ \\
\hline Septal annular systolic velocity (cm/s) & 8.4 & 1.0 & 6,12 & 8.0 & 1.0 & 6,11 & 0.01 \\
\hline Lateral annular E' (cm/s) & 14.7 & 2.7 & 10,23 & 15.0 & 2.5 & 9,20 & NS \\
\hline Lateral annular A' (cm/s) & 6.8 & 1.4 & 4,11 & 6.6 & 1.2 & 4,9 & NS \\
\hline Lateral annular E'/A' & 2.3 & 0.7 & $1.1,3.8$ & 2.3 & 0.6 & $1.3,4$ & NS \\
\hline Lateral annular E/E' & 5.1 & 1.0 & $2.3,7.8$ & 5.8 & 1.2 & $3.3,9.8$ & $<0.001$ \\
\hline Lateral annular systolic velocity (cm/s) & 10.3 & 2.0 & 6,15 & 10.0 & 1.6 & 7,14 & NS \\
\hline GLS (\%) & -18.7 & 1.6 & $-16,-22$ & -20.7 & 2.1 & $-15,-24$ & $<0.001$ \\
\hline SoV diameter (mm) & 32.6 & 3.5 & 26,40 & 27.5 & 2.5 & 22,35 & $<0.001$ \\
\hline SoV diameter/BSA (mm/m²) & 17.4 & 1.9 & $13.7,21.4$ & 16.7 & 1.8 & $12.6,21.6$ & 0.02 \\
\hline \multicolumn{8}{|l|}{ Right heart } \\
\hline RA volume (ml) & 64.6 & 23.4 & 25,155 & 44.1 & 13.9 & 25,95 & $<0.001$ \\
\hline RA volume/BSA (ml/m²) & 34.4 & 12.3 & $14.1,84$ & 26.6 & 7.8 & $15.2,52.4$ & $<0.001$ \\
\hline RVOTpl (mm) & 29.6 & 3.7 & 22,40 & 27.2 & 4.0 & 16,36 & $<0.001$ \\
\hline RVOTp (mm) & 33.5 & 4.7 & 22,46 & 30.5 & 5.2 & 20,49 & $<0.001$ \\
\hline RVOTd (mm) & 23.8 & 2.9 & 15,30 & 21.9 & 2.9 & 14,30 & $<0.001$ \\
\hline RVD basal (mm) & 39.2 & 5.4 & 14,54 & 35.1 & 3.8 & 26,44 & $<0.001$ \\
\hline RVD mid (mm) & 34.4 & 4.2 & 26,50 & 30.6 & 4.2 & 20,42 & $<0.001$ \\
\hline RVD longitudinal (mm) & 80.6 & 7.2 & 64,97 & 71.6 & 8.0 & 51,91 & $<0.001$ \\
\hline TAPSE (mm) & 25.1 & 3.4 & 19,37 & 24.4 & 3.0 & 18,32 & NS \\
\hline RV E' (cm/s) & 12.6 & 2.3 & 7,18 & 12.9 & 2.0 & 9,19 & NS \\
\hline $\mathrm{RV} \mathrm{A}^{\prime}(\mathrm{cm} / \mathrm{s})$ & 8.8 & 2.3 & 3,16 & 8.6 & 2.4 & 5,15 & NS \\
\hline$R V E^{\prime} / A^{\prime}$ & 1.5 & 0.5 & $0.8,3.7$ & 1.6 & 0.5 & $0.7,3.2$ & NS \\
\hline RV sys vel (cm/s) & 12.2 & 1.8 & 8,19 & 11.6 & 1.4 & 9,15 & 0.02 \\
\hline
\end{tabular}


TABLE 3 | Continued

\begin{tabular}{|c|c|c|c|c|c|}
\hline LV geometry & $\begin{array}{l}\text { No. of } \\
\text { athletes }\end{array}$ & $\%$ & $\begin{array}{c}\text { No. of } \\
\text { athletes }\end{array}$ & $\%$ & \\
\hline Normal & 54 & (72) & 62 & (82.7) & NS \\
\hline Concentric remodeling & 10 & (13.3) & 3 & (4) & \\
\hline Eccentric hypertrophy & 8 & $(10.7)$ & 8 & $(10.7)$ & \\
\hline Concentric hypertrophy & 3 & (4) & 2 & $(2.7)$ & \\
\hline
\end{tabular}

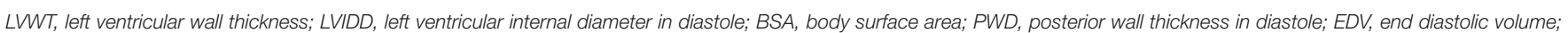

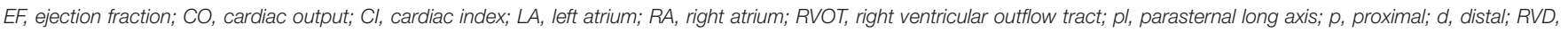
right ventricular diameter; DT, deceleration time; TAPSE, tricuspid annular plane systolic excursion; GLS, global longitudinal strain; SoV, Sinus of Valsalva.

\section{Echocardiographic Data}

Comprehensive structural and functional echocardiographic parameters for the cohort by gender and sport discipline are presented in Tables 3, 4, respectively. Normal geometry was encountered in the majority of athletes $(77.3 \%, n=116)$, followed by eccentric hypertrophy $(n=16,10.7 \%)$, concentric remodeling $(n=13,8.7 \%)$, and concentric hypertrophy $(n=$ $5,3.3 \%)$.

Of the entire cohort, 3 athletes (2\% prevalence) were found to have minor structural heart disease that did not impact sporting participation: secundum atrial septal defect (male kayaker), dilated aortic root (male cyclist), and anterior mitral valve leaflet prolapse with mild mitral regurgitation (female triathlete).

\section{Differences Between Genders}

Absolute LV wall thickness (LVWT), LVIDD, volumes and mass were significantly larger in male compared to female athletes (Table 3 and Figure 1). In male athletes, diastolic indices, and global longitudinal strain were of smaller magnitudes compared to female athletes. There were no differences between male and female athletes in terms of LV geometry.

\section{Differences Between Sport Disciplines}

Athletes from mixed or endurance sports demonstrated significantly increased left and right heart dimensions (i.e., LVWT, LVIDD, LV mass, LV end diastolic volume, LV stroke volume, left and right atrial volumes) compared to those from skill or power-based sports. Diastolic function and global longitudinal strain did not differ significantly between the two groups (Table 4).

\section{Differences Between Ethnic Groups}

Echocardiographic parameters in Chinese and non-Chinese athletes were comparable for almost all variables.

\section{Regression Analyses}

Logistic regression analysis showed that increasing age was an independent predictor for ATWI beyond V2 (odds ratio 1.13, $p=0.03$ ) after adjusting for baseline demographics (age, gender, ethnicity, type of sport, training hours). Linear regression analysis showed that male gender and mixed and endurance sport disciplines were independently associated with a statistically significantly higher LVIDD, LVWT, and LV end diastolic volume after adjusting for baseline demographics.

\section{DISCUSSION}

This prospective electrocardiographic and echocardiographic registry is the first to evaluate a well-defined cohort of SE Asian elite athletes. It utilizes the contemporary IR2017 and describes a comprehensive range of ECG and echocardiographic characteristics, including values indexed to body surface area. Notably, the prevalence of ATWI beyond lead V2 on ECG was noted to be high at $9.3 \%$ for female athletes.

\section{Electrocardiographic Parameters Comparison With Other Athletic Cohorts}

In non-Asian athletes, evolution of ECG interpretation criteria has led to considerable reduction in prevalence of abnormal ECGs while preserving sensitivity for detection of pathology. Prior to the current gold standard IR2017, its predecessor the RC2014 was used to compare athletes from different ethnicities. Reported prevalence rates of abnormal ECGs in Black, Caucasian and Arabic athletes using RC2014 (11.5, 5.3, and $3.6 \%$, respectively) were substantially lower than those with ESC2010 and SC2013 (10, 15). Likewise, our cohort mirrors this trend of reduction in abnormal ECG with application of each newer criterion (Figure 2).

Application of IR2017 in a cohort of more than 11,000 adolescent soccer players revealed prevalence rates of abnormal ECGs at 3.6 and $1.6 \%$ for black and white athletes, respectively (16). Comparatively, the prevalence of abnormal ECGs in our cohort did not reduce further from RC2014 to IR2017 largely because our population was older in age and unaffected by the inclusion of the juvenile ECG pattern in IR2017 (17).

At $6.7 \%$, the prevalence of abnormal ECGs in our SE Asian athletes is higher than reported figures for most non-Asian athletic populations. This is accounted for predominantly by ATWI beyond lead V2. Specifically, ATWI beyond V2 amongst SE Asian female athletes has a prevalence of $9.3 \%$, more than 4 times the corresponding prevalence of $2.1 \%$ in a large cohort of Caucasian female athletes from UK (18). 
TABLE 4 | Differences in echocardiographic parameters of Singapore athletes based on sporting discipline.

\begin{tabular}{|c|c|c|c|c|c|}
\hline & \multicolumn{2}{|c|}{$\begin{array}{l}\text { Skill and power } \\
\qquad(n=44)\end{array}$} & \multicolumn{2}{|c|}{$\begin{array}{l}\text { Mixed and endurance } \\
\qquad(n=106)\end{array}$} & \multirow[t]{2}{*}{$P$ value } \\
\hline & Mean & $S D$ & Mean & $S D$ & \\
\hline \multicolumn{6}{|l|}{ Left heart } \\
\hline LVWT (mm) & 7.8 & 1.3 & 8.9 & 1.4 & $<0.001$ \\
\hline LVWT/BSA (mm/m²) & 4.5 & 0.6 & 5.0 & 0.8 & $<0.001$ \\
\hline LVIDD (mm) & 48.1 & 4.1 & 50.3 & 4.0 & 0.004 \\
\hline LVIDD/BSA $\left(\mathrm{mm} / \mathrm{m}^{2}\right)$ & 27.6 & 2.4 & 28.6 & 2.7 & 0.025 \\
\hline LVPWD (mm) & 8.0 & 1.3 & 9.1 & 1.4 & $<0.001$ \\
\hline Relative wall thickness & 0.33 & 0.05 & 0.36 & 0.05 & 0.002 \\
\hline LV mass $(\mathrm{g})$ & 127.3 & 39.8 & 162.3 & 47.1 & $<0.001$ \\
\hline LV mass/BSA $\left(\mathrm{g} / \mathrm{m}^{2}\right)$ & 71.9 & 17.3 & 91.3 & 23.0 & $<0.001$ \\
\hline LVEDV (ml) & 98.2 & 24.3 & 116.0 & 26.2 & $<0.001$ \\
\hline LVEDV/BSA $\left(\mathrm{ml} / \mathrm{m}^{2}\right)$ & 55.2 & 8.8 & 65.5 & 12.7 & $<0.001$ \\
\hline LV stroke volume (ml) & 58.0 & 13.7 & 68.2 & 16.1 & $<0.001$ \\
\hline LVEF (\%) & 59.5 & 4.0 & 59.0 & 4.2 & NS \\
\hline $\mathrm{LA}(\mathrm{mm})$ & 32.3 & 4.3 & 35.5 & 4.2 & $<0.001$ \\
\hline LA/BSA $\left(\mathrm{mm} / \mathrm{m}^{2}\right)$ & 18.5 & 1.7 & 20.2 & 2.4 & $<0.001$ \\
\hline LA volume (ml) & 48.3 & 14.3 & 63.7 & 17.2 & $<0.001$ \\
\hline LA volume/BSA $\left(\mathrm{ml} / \mathrm{m}^{2}\right)$ & 27.3 & 6.3 & 36.0 & 8.7 & $<0.001$ \\
\hline E velocity $(\mathrm{cm} / \mathrm{s})$ & 81.3 & 18.3 & 78.7 & 14.6 & NS \\
\hline DT (ms) & 153.9 & 19.5 & 153.4 & 20.2 & NS \\
\hline A velocity $(\mathrm{cm} / \mathrm{s})$ & 42.7 & 9.2 & 40.9 & 8.9 & NS \\
\hline E/A & 2.0 & 0.6 & 2.0 & 0.5 & NS \\
\hline Septal annular E' (cm/s) & 12.1 & 2.0 & 11.7 & 1.7 & NS \\
\hline Septal annular A' (cm/s) & 7.1 & 1.5 & 6.7 & 1.4 & NS \\
\hline Septal annular E'/A' & 1.8 & 0.5 & 1.8 & 0.5 & NS \\
\hline Septal annular E/E' & 6.7 & 1.5 & 6.8 & 1.4 & NS \\
\hline $\begin{array}{l}\text { Septal annular systolic } \\
\text { velocity }(\mathrm{cm} / \mathrm{s})\end{array}$ & 8.2 & 1.0 & 8.3 & 1.0 & NS \\
\hline $\begin{array}{l}\text { Lateral annular E' } \\
(\mathrm{cm} / \mathrm{s})\end{array}$ & 15.1 & 2.5 & 14.7 & 2.6 & NS \\
\hline $\begin{array}{l}\text { Lateral annular } A^{\prime} \\
(\mathrm{cm} / \mathrm{s})\end{array}$ & 6.6 & 1.4 & 6.7 & 1.3 & NS \\
\hline Lateral annular E'/A' & 2.4 & 0.6 & 2.3 & 0.6 & NS \\
\hline Lateral annular E/E' & 5.4 & 1.4 & 5.4 & 1.1 & NS \\
\hline $\begin{array}{l}\text { Lateral annular systolic } \\
\text { velocity }(\mathrm{cm} / \mathrm{s})\end{array}$ & 10.6 & 1.7 & 9.9 & 1.8 & 0.038 \\
\hline GLS (\%) & -20.0 & 2.2 & -19.6 & 2.0 & NS \\
\hline SoV diameter (mm) & 29 & 3.8 & 30.5 & 3.9 & NS \\
\hline $\begin{array}{l}\text { SoV diameter/BSA } \\
\left(\mathrm{mm} / \mathrm{m}^{2}\right)\end{array}$ & 16.6 & 1.6 & 17.3 & 1.9 & NS \\
\hline \multicolumn{6}{|l|}{ Right heart } \\
\hline RA volume (ml) & 42.9 & 15.7 & 59.1 & 22.3 & $<0.001$ \\
\hline $\begin{array}{l}\text { RA volume/BSA } \\
\left(\mathrm{ml} / \mathrm{m}^{2}\right)\end{array}$ & 24.0 & 6.5 & 33.2 & 11.3 & $<0.001$ \\
\hline RVOTpl (mm) & 26.5 & 4.4 & 29.2 & 3.6 & 0.001 \\
\hline RVOTp (mm) & 30.3 & 6.2 & 32.7 & 4.6 & 0.024 \\
\hline RVOTd (mm) & 21.7 & 3.0 & 23.3 & 3.0 & 0.006 \\
\hline RVD basal (mm) & 35.1 & 4.2 & 37.9 & 5.2 & 0.001 \\
\hline RVD mid (mm) & 31.1 & 4.0 & 33.1 & 4.7 & 0.01 \\
\hline
\end{tabular}

(Continued)
TABLE 4 | Continued

\begin{tabular}{|c|c|c|c|c|c|}
\hline & \multicolumn{2}{|c|}{$\begin{array}{l}\text { Skill and power } \\
\qquad(n=44)\end{array}$} & \multicolumn{2}{|c|}{$\begin{array}{l}\text { Mixed and endurance } \\
\qquad(n=106)\end{array}$} & \multirow[t]{2}{*}{$P$ value } \\
\hline & Mean & $S D$ & Mean & $S D$ & \\
\hline RVD longitudinal (mm) & 74.3 & 9.4 & 76.9 & 8.5 & NS \\
\hline TAPSE (mm) & 23.9 & 2.9 & 25.1 & 3.3 & 0.033 \\
\hline $\mathrm{RV}^{\prime}$ (cm/s) & 13.0 & 2.1 & 12.6 & 2.2 & NS \\
\hline $\mathrm{RV} \mathrm{A}^{\prime}(\mathrm{cm} / \mathrm{s})$ & 8.3 & 2.1 & 8.9 & 2.4 & NS \\
\hline$R V E^{\prime} / A^{\prime}$ & 1.6 & 0.5 & 1.5 & 0.5 & NS \\
\hline RV sys vel $(\mathrm{cm} / \mathrm{s})$ & 11.7 & 1.6 & 12.0 & 1.7 & NS \\
\hline
\end{tabular}

LWWT, left ventricular wall thickness; LVIDD, left ventricular internal diameter in diastole; $B S A$, body surface area; PWD, posterior wall thickness in diastole; EDV, end diastolic volume; EF, ejection fraction; $C O$, cardiac output; $C l$, cardiac index; $L A$, left atrium; RA, right atrium; RVOT, right ventricular outflow tract; pl, parasternal long axis; $p$, proximal; d, distal; RVD, right ventricular diameter; DT, deceleration time; TAPSE, tricuspid annular plane systolic excursion; GLS, global Iongitudinal strain; SoV, Sinus of Valsalva.

Whereas, ATWI in non-Asian athletes may prompt further evaluation for possible cardiomyopathy (e.g., hypertrophic cardiomyopathy or arrhythmogenic cardiomyopathy), the incidence of pathological conditions manifesting with ATWI is very low in Singapore (19). For instance, the prevalence of hypertrophic cardiomyopathy in a large unselected young Singapore male population was $0.005 \%$ (20). In addition, incidence of sports-related sudden death was $<1$ in 2 million (21). These data may suggest a physiological rather than pathological explanation for the high prevalence of ATWI. A possible hypothesis is the displacement of the cardiac apex following longstanding endurance activity, which may be further influenced by unique contributions from gender and ethnicity (22).

The combined impact of ethnicity, gender, and sport discipline on ATWI deserves validation in a larger cohort. Confirmation of the high prevalence of ATWI up to lead $\mathrm{V} 3$ in Asian female endurance athletes combined with normal cardiac function and structure may lead to further refinement of ECG interpretation criteria with additional ethnic-specific cutoffs.

\section{Echocardiographic Parameters}

Our study identified gender differences between athletes in global longitudinal strain, where male athletes have reduced GLS compared to females (an absolute difference of 2\%). These gender differences corroborate findings by Park et al. in their study of an international cohort of university athletes and provide further insights into gender disparities in physiological cardiac remodeling (23).

\section{Comparison With Other Athletic Cohorts}

Compared to Caucasian and Black athletes, both male and female SE Asian athletes had smaller absolute LVIDD and LVWT $(5,6,24-26)$ (Figure 3). A likely explanation for this is the proportionately smaller body sizes of SE Asians in general. However, the opposite was true when indexed values were used. Amongst male athletes, indexing of LVIDD to 


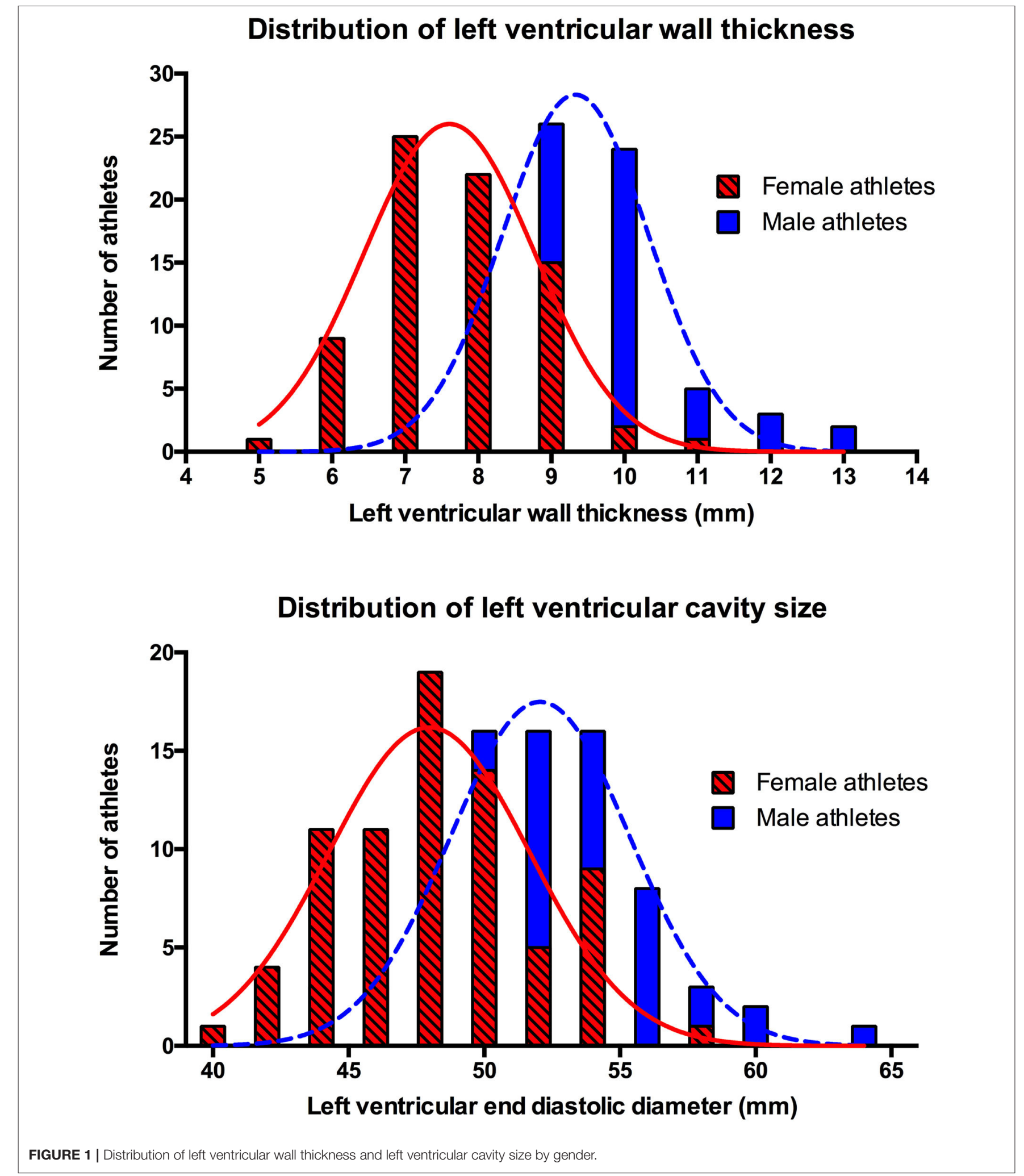

body surface area revealed that a cohort of Caucasian and Black professional basketball players from the United States had smaller dimensions compared to our cohort (25). This may be due to the majority (more than 40\%) of our cohort consisting of endurance athletes. Athletes from endurance sports typically develop larger LVIDD compared to strength-trained 


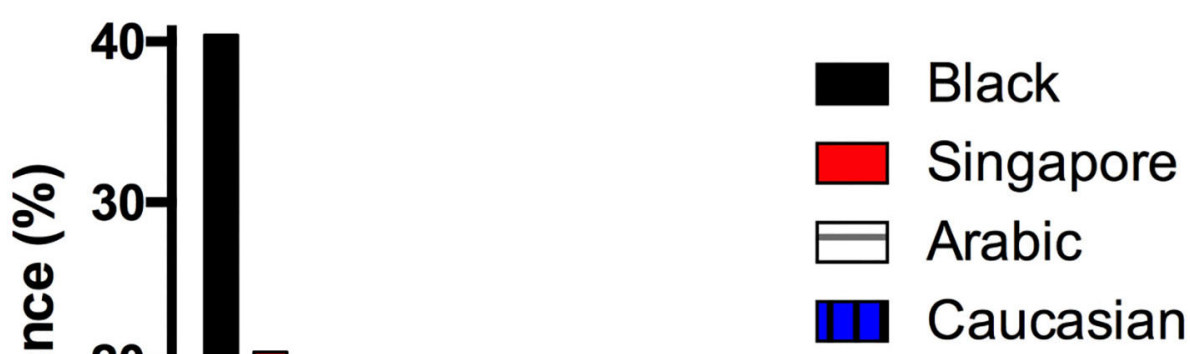

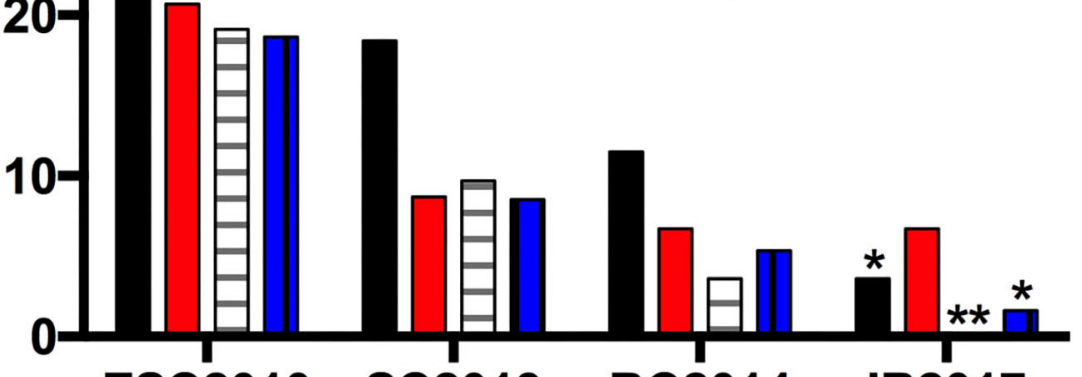 \\ $\begin{array}{llll}\text { ESC2010 } & \text { SC2013 } & \text { RC2014 } & \text { IR2017 }\end{array}$ \\ Athlete ECG Interpretation Criteria}

FIGURE 2 | Prevalence of abnormal electrocardiograms in athletes of different ethnicities $(10,15,16)\left({ }^{*}\right.$ Adolescent athletes; **Data unavailable; ESC, European Society of Cardiology; SC, Seattle Criteria; RC, Refined Criteria; IR, International Recommendations).

athletes or those engaged in mixed sport such as basketball $(27,28)$.

Our SE Asian athlete cohort LVIDD and LVWT values corroborate with a group of Singapore athletes studied in 2004 and a group of mixed Asian university athletes in 2015 $(29,30)$. However, when compared to athletes from China and Japan, varying limits were observed with no discernable pattern identified. Male athletes from China and Japan had larger absolute LVIDD than those from our cohort. Nonetheless, this pattern was no longer present after indexing to body surface area $(31,32)$. China athletes also had higher absolute LVWT compared to our cohort, although the difference between cohorts was reversed after indexing for body surface area.

These differences between varying Asian cohorts underscore the impact of ethnicity on cardiac remodeling in athletes. Riding et al. demonstrated substantial variability in electrical and structural remodeling amongst Black athletes from different parts of the world, highlighting the influence of geographic origin (33). Their findings mirror our comparisons between SE Asian athletes and other East Asian cohorts. This suggests that, despite sharing a common ancestry, Asian athletes from different geographic locations have intrinsic differences in physiological electrical and structural cardiac remodeling. These differences warrant additional study in other Asian cohorts such as Malay, Thai, Filipino and Indonesian athletes, to avoid generalization of findings for all athletes of Asian descent.

Moreover, comparison of echocardiographic parameters indexed to body surface area is challenging as these are uncommonly reported in existing athlete cohorts. We encourage researchers involved in athlete registries to include indexed values to facilitate comparison of echocardiographic parameters.

\section{Limitations}

Our registry of Singapore athletes has a sample size of 150 with predominant Chinese ethnicity, limiting applicability to all Asian athletes. Although nonChinese athletes make up $12 \%$ of the cohort, these ethnic groups (Malay, Indian, Sikh, Indonesian) reflect the multi-racial population in Singapore and SE Asia. Importantly, comparison between Chinese and nonChinese athletes did not reveal any clinically significant differences in demographics, prevalence of abnormal ECG or echocardiographic parameters. Within our cohort, representation from athletes engaging in power-based sports is also limited, with power-trained athletes making up $16 \%$ of the cohort.

Not all athletes with abnormal ECGs received comprehensive evaluation beyond echocardiography, for instance cardiac magnetic resonance imaging, exercise testing or $24 \mathrm{~h}$ electrocardiographic monitoring, to definitively exclude cardiac pathology. However, none of the athletes with abnormal ECGs were noted to have abnormal symptoms, examination findings or unexplained drop in fitness during their routine 2 -yearly pre-competition clinical evaluations to date.

Given the small number of athletes with ATWI, regression analyses should be interpreted with caution. 


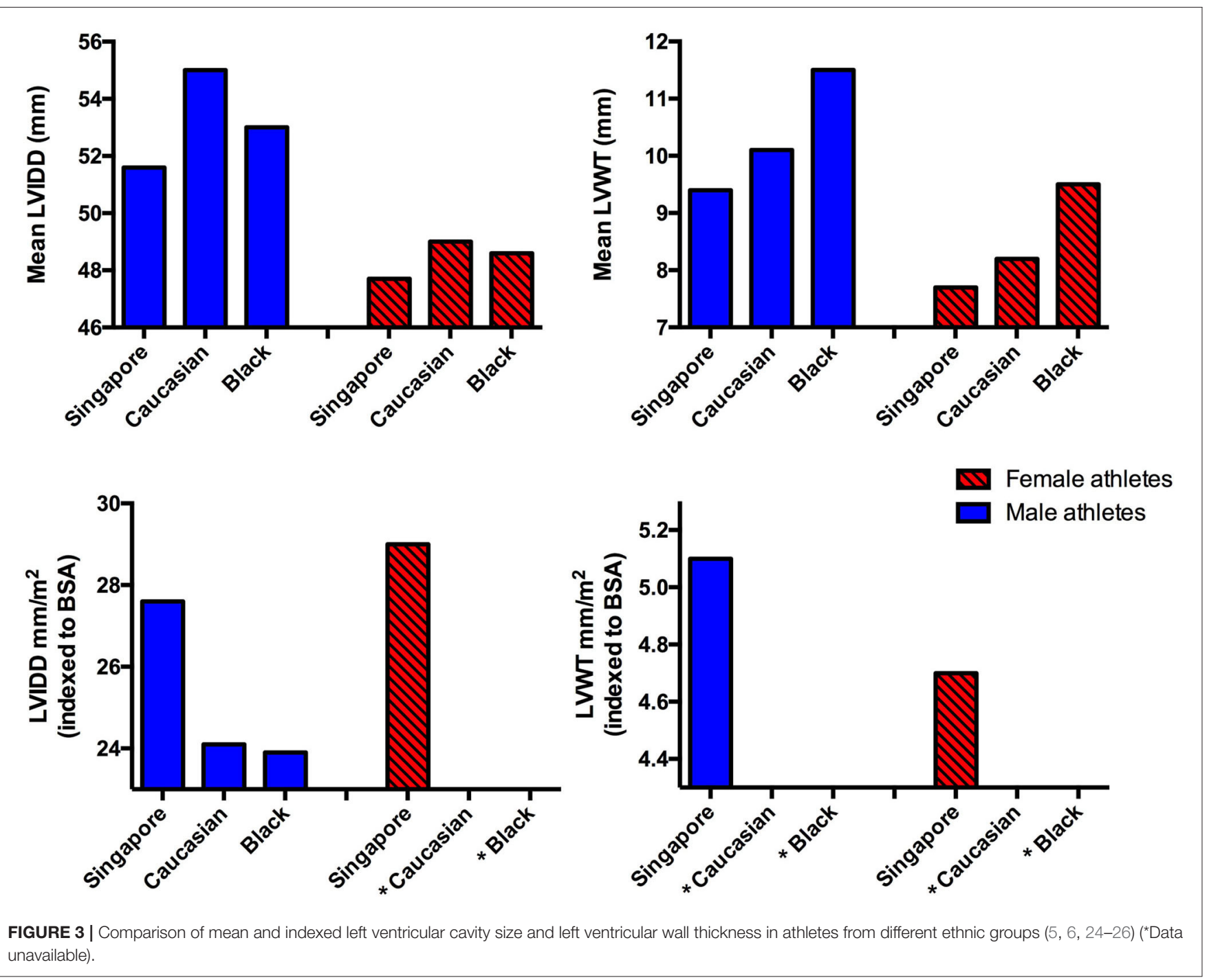

Finally, we did not perform comparison with matched Caucasian and/or Black athletes to highlight ethnic-specific differences in ECG and echocardiographic characteristics.

\section{CONCLUSION}

This prospective sports cardiology registry highlights electrocardiographic and echocardiographic characteristics in a cohort of SE Asian elite athletes as well as differences between other Asian and non-Asian cohorts. Further large-scale prospective registries are necessary to identify aspects of cardiac remodeling unique to athletes of different ethnic backgrounds.

\section{DATA AVAILABILITY STATEMENT}

The original contributions presented in the study are included in the article/Supplementary Materials, further inquiries can be directed to the corresponding authors.

\section{ETHICS STATEMENT}

This study was reviewed and approved by National Healthcare Group Domain Specific Review Board Singapore (2017/00319) and Singapore Sports Institute Institutional Review Board (PH/EXP/018). All participants provided their written informed consent to participate in this study.

\section{AUTHOR CONTRIBUTIONS}

LK performed the measurements. MW, RG, FT, and GC were involved in planning and recruitment of participants. TY processed the experimental data, performed the analysis, drafted the manuscript, and designed the figures. NT edited the manuscript. S-PC aided in statistical analysis and interpreting the results. JM, C-HL, DO, AM, SS, and AR provided critical feedback and helped shape the research, analysis, and manuscript. All authors discussed the results and contributed to the final manuscript. 


\section{FUNDING}

This study was supported by a National University Health System Clinician Scientist Program award (N-171-000-469-001), and a centre grant (CGAug16M008) from the National Medical Research Council.

\section{ACKNOWLEDGMENTS}

We would like to acknowledge all the Singapore national athletes who have contributed their time to participate in

\section{REFERENCES}

1. Noland M, Stahler K. Asian participation and performance at the olympic games. Asian Econ Policy Rev. (2016) 11:70-90. doi: 10.1111/aepr.12118

2. Wikipedia. Asia. Available online at: https://en.wikipedia.org/wiki/Asia (accessed August 01, 2021)

3. Yeo TJ, Sharma S. Using the 12-lead electrocardiogram in the care of athletic patients. Cardiol Clin. (2016) 34:543-55. doi: 10.1016/j.ccl.2016.06.004

4. Baggish AL. A decade of athlete ECG criteria: where we've come and where we're going. J Electrocardiol. (2015) 48:324-8. doi: 10.1016/j.jelectrocard.2015.02.002

5. Pelliccia A, Caselli S, Sharma S, Basso C, Bax JJ, Corrado D, et al. European Association of Preventive Cardiology (EAPC) and European Association of Cardiovascular Imaging (EACVI) joint position statement: recommendations for the indication and interpretation of cardiovascular imaging in the evaluation of the athlete's heart. Eur Heart J. (2018) 39:1949-69. doi: 10.1093/eurheartj/ehx532

6. Oxborough D, Augustine D, Gati S, George K, Harkness A, Mathew T, et al. A guideline update for the practice of echocardiography in the cardiac screening of sports participants: a joint policy statement from the British Society of Echocardiography and Cardiac Risk in the Young. Echo Res Pract. (2018) 5:G1-G10. doi: 10.1530/ERP-17-0075

7. Wikipedia. Singapore. Available online at: https://en.wikipedia.org/wiki/ Singapore (accessed August 01, 2021).

8. Corrado D, Pelliccia A, Heidbuchel H, Sharma S, Link M, Basso C, et al. Recommendations for interpretation of 12-lead electrocardiogram in the athlete. Eur Heart J. (2010) 31:243-59. doi: 10.1093/eurheartj/ehp473

9. Drezner JA, Ackerman MJ, Anderson J, Ashley E, Asplund CA, Baggish AL, et al. Electrocardiographic interpretation in athletes: the 'Seattle criteria'. $\mathrm{Br} \mathrm{J}$ Sports Med. (2013) 47:122-4. doi: 10.1136/bjsports-2012-092067

10. Sheikh N, Papadakis M, Ghani S, Zaidi A, Gati S, Adami PE, et al. Comparison of electrocardiographic criteria for the detection of cardiac abnormalities in elite black and white athletes. Circulation. (2014) 129:163749. doi: 10.1161/CIRCULATIONAHA.113.006179

11. Sharma S, Drezner JA, Baggish A, Papadakis M, Wilson MG, Prutkin JM, et al. International recommendations for electrocardiographic interpretation in athletes. Eur Heart J. (2018) 39:1466-80. doi: 10.1093/eurheartj/ehw631

12. Lang RM, Badano LP, Mor-Avi V, Afilalo J, Armstrong A, Ernande L, et al. Recommendations for cardiac chamber quantification by echocardiography in adults: an update from the American Society of Echocardiography and the European Association of Cardiovascular Imaging. J Am Soc Echocardiogr. (2015) 28:1-39.e14. doi: 10.1016/j.echo.2014.10.003

13. Wharton G, Steeds R, Allen J, Phillips H, Jones R, Kanagala P, et al. A minimum dataset for a standard adult transthoracic echocardiogram: a guideline protocol from the British Society of Echocardiography. Echo Res Pract. (2015) 2:G9-G24. doi: 10.1530/ERP-14-0079

14. Lang RM, Bierig M, Devereux RB, Flachskampf FA, Foster E, Pellikka PA, et al. Recommendations for chamber quantification. Eur J Echocardiogr. (2006) 7:79-108. doi: 10.1016/j.euje.2005.12.014

15. Riding NR, Sheikh N, Adamuz C, Watt V, Farooq A, Whyte GP, et al. Comparison of three current sets of electrocardiographic interpretation this Sports Cardiology registry, as well as colleagues who have contributed their valuable time and effort to assist with recruitment.

\section{SUPPLEMENTARY MATERIAL}

The Supplementary Material for this article can be found online at: https://www.frontiersin.org/articles/10.3389/fcvm. 2021.799129/full\#supplementary-material criteria for use in screening athletes. Heart. (2015) 101:384-90. doi: 10.1136/heartjnl-2014-306437

16. Malhotra A, Dhutia H, Yeo T-J, Finocchiaro G, Gati S, Bulleros P, et al. Accuracy of the 2017 international recommendations for clinicians who interpret adolescent athletes' ECGs: a cohort study of 11168 British white and black soccer players. Br J Sports Med. (2020) 54:739-45. doi: 10.1136/bjsports-2017-098528

17. Basu J, Malhotra A. Interpreting the Athlete's ECG: current state and future perspectives. Curr Treat Options Cardiovasc Med. (2018) 20:104 doi: 10.1007/s11936-018-0693-0

18. Malhotra A, Dhutia H, Gati S, Yeo TJ, Dores H, Bastiaenen R, et al. Anterior T-Wave inversion in young white athletes and nonathletes: prevalence and significance. J Am Coll Cardiol. (2017) 69:1-9. doi: 10.1016/j.jacc.2016. 10.044

19. Leischik R, Dworrak B, Strauss M, et al. Special Article - Exercise-induced right ventricular injury or arrhythmogenic cardiomyopathy (ACM): the bright side and the dark side of the moon. Prog Cardiovasc Dis. (2020) 63:671-81. doi: 10.1016/j.pcad.2020.03.015

20. Ng CT, Chee TS, Ling LF, Lee YP, Ching CK, Chua TS, et al. Prevalence of hypertrophic cardiomyopathy on an electrocardiogram-based preparticipation screening programme in a young male South-East Asian population: results from the Singapore Armed Forces Electrocardiogram and Echocardiogram screening protocol. Europace. (2011) 13:883-8. doi: 10.1093/europace/eur051

21. Chao TC. Death in sports and recreation. Ann Acad Med Singap. (1983) 12:400-4.

22. Brosnan MJ, Claessen G, Heidbuchel H, Prior DL, La Gerche A. Right Precordial T-Wave Inversion in healthy endurance athletes can be explained by lateral displacement of the cardiac apex. JACC Clin Electrophysiol. (2015) 1:84-91. doi: 10.1016/j.jacep.2015.03.007

23. Park JH, Oh JK, Kim KH, Cho JY, Cho GY, Lee JH, et al. Left ventricular longitudinal strain and strain rate values according to sex and classifications of sports in the young university athletes who participated in the 2015 Gwangju Summer Universiade. JACC Cardiovasc Imaging. (2018) 11:1719-21. doi: 10.1016/j.jcmg.2018.02.012

24. Galderisi M, Cardim N, D’Andrea A, Bruder O, Cosyns B, Davin L, et al. The multi-modality cardiac imaging approach to the Athlete's heart: an expert consensus of the European Association of Cardiovascular Imaging. Eur Heart J Cardiovasc Imaging. (2015) 16:353. doi: 10.1093/ehjci/j eu323

25. Engel DJ, Schwartz A, Homma S. Athletic cardiac remodeling in US professional basketball players. JAMA Cardiol. (2016) 1:80-7. doi: 10.1001/jamacardio.2015.0252

26. Sheikh N, Sharma S. Impact of ethnicity on cardiac adaptation to exercise. Nat Rev Cardiol. (2014) 11:198-217. doi: 10.1038/nrcardio.2 014.15

27. Weiner RB, Baggish AL. Exercise-induced cardiac remodeling. Prog Cardiovasc Dis. (2012) 54:380-6. doi: 10.1016/j.pcad.2012.01.006

28. Morganroth J, Maron BJ, Henry WL, Epstein SE. Comparative left ventricular dimensions in trained athletes. Ann Intern Med. (1975) 82:521-4. doi: $10.7326 / 0003-4819-82-4-521$ 
29. Keh YS, Tan PJ, Chai SC, Tan B, Tong KL. Physiologic limits of cardiac remodelling in Asian competitive athletes - a single centre study. Ann Acad Med Singap. (2018) 47:230-2.

30. Cho JY, Kim KH, Rink L, Hornsby K, Park H, Park JH, et al. University athletes and changes in cardiac geometry: insight from the 2015 Gwangju Summer Universiade. Eur Heart J Cardiovasc Imaging. (2019) 20:407-16. doi: 10.1093/ehjci/jey196

31. Sun B, Ma JZ, Yong YH, Lv YY. The upper limit of physiological cardiac hypertrophy in elite male and female athletes in China. Eur J Appl Physiol. (2007) 101:457-63. doi: 10.1007/s00421-007-0517-5

32. Kervio G, Pelliccia A, Nagashima J, Wilson MG, Gauthier J, Murayama $M$, et al. Alterations in echocardiographic and electrocardiographic features in Japanese professional soccer players: comparison to African-Caucasian ethnicities. Eur J Prev Cardiol. (2013) 20:880-8. doi: 10.1177/2047487312447905

33. Riding NR, Sharma S, McClean G, Adamuz C, Watt V, Wilson MG. Impact of geographical origin upon the electrical and structural manifestations of the black athlete's heart. Eur Heart J. (2019) 40:50-8. doi: 10.1093/eurheartj/ehy521
Conflict of Interest: The authors declare that the research was conducted in the absence of any commercial or financial relationships that could be construed as a potential conflict of interest.

Publisher's Note: All claims expressed in this article are solely those of the authors and do not necessarily represent those of their affiliated organizations, or those of the publisher, the editors and the reviewers. Any product that may be evaluated in this article, or claim that may be made by its manufacturer, is not guaranteed or endorsed by the publisher.

Copyright (c) 2022 Yeo, Wang, Grignani, McKinney, Koh, Tan, Chan, Tay, Chan, Lee, Oxborough, Malhotra, Sharma and Richards. This is an open-access article distributed under the terms of the Creative Commons Attribution License (CC BY). The use, distribution or reproduction in other forums is permitted, provided the original author(s) and the copyright owner(s) are credited and that the original publication in this journal is cited, in accordance with accepted academic practice. No use, distribution or reproduction is permitted which does not comply with these terms. 